\title{
Oncological Rehabilitation and Cancer Survivorship
}

\author{
Oliver Rick ${ }^{a}$ Thorsten Langer ${ }^{b}$ \\ ${ }^{a}$ Klinik Reinhardshöhe, Bad Wildungen, Germany; \\ bPädiatrische Onkologie und Hämatologie, Klinik für Kinder- und Jugendmedizin, Universitätsklinikum Schleswig-Holstein, Lübeck, Germany
}

In Germany every year more than 470,000 people suffer from cancer. About 25,000 patients are younger than 40 years of age at diagnosis of cancer and about 2,000 are younger than 18 years of age. Many of them will not die from cancer - as survival rates are rising up to $80 \%$, the group of long-term cancer survivors grows. However, data from the United States show that 30 years after cancer treatment $2 / 3$ of the survivors suffer from long-term complications. In this issue of Oncology Research and Treatment, we will discuss the problems and challenges that long-term cancer survivors face as well as the possibilities of rehabilitation to lessen this burden.

In the manuscript by Langer et al. [1] long-term complications after childhood cancer are described and models of care for the transition from pediatric oncology to internal medicine are discussed. Since the 1980 family-oriented oncological rehabilitation (FOR) is a fixed component of the pediatric oncological treatment strategies. Krauth [2] reports on the oncological rehabilitation of children, adolescents, and young adults (AYA) and the relevance in the long-term follow-up.

Over the last 20 years, oncological rehabilitation has evolved to become an integral part of our health care system. The costs of this measure are mainly borne by the German Pension Insurance (DRV, 'Deutsche Rentenversicherung') and by the health insurance companies. Currently, nearly 160,000 cancer patients benefit from this therapeutic option at the expense of the DRV to improve their medical condition. Oncological rehabilitation has thus been given high priority in cancer care and is seamlessly integrated with acute medical care. The manuscript by Rick et al. [3] describes in detail the history of rehabilitation and its special position in our social system. In addition, the author describes the objectives and global content of oncological rehabilitation and provides an overview of the current evidence.

The manuscript by Schmielau et al. [4] is dedicated to the longterm toxicities after cancer treatment. These often considerably impact the patient's social and professional life and thus represent a significant treatment emphasis in oncological rehabilitation. Therefore, oncological rehabilitation should be considered as a starting point of cancer survivorship, as it addresses problems from a holis- tic aspect at an early stage after completion of cancer therapy. In addition to the psychological consequences of cancer, the focus is set on somatic disorders as long-term consequences of cancer treatment, such as cytotoxic-induced polyneuropathy, cognitive dysfunction, chronic fatigue, impairment of sexuality and fertility as well as cardiotoxicities. The authors point out in their contribution the importance of these disorders for the patients, as well as the therapeutic options in the context of cancer survivorship.

The manuscript by Seifart and Schmielau [5] describes the importance of oncological rehabilitation for employment. Although approximately $70 \%$ of patients return to work, many of them are affected by changes at the workplace and financial losses. Patients often experience the financial burden as heavier than the physical and psychological consequences of cancer. As a result, there is further strain on the social and psychological situation, the development of poverty, which leads to a decrease in participation in community life and an increase in mortality. In addition to the severe individual consequences for the patient, this will also incur costs for employers and the social insurance funds. These include costs for the treatment of mental suffering, long periods of incapacity to work, and the partial or complete withdrawal from the labor market, which results in drawing a full-rate reduced earning capacity pension. This shall be avoided by using the instruments for participation in working life (LTAs), mainly funded by the DRV, and the possibilities offered by oncological rehabilitation.

The reviews in this issue of ONCOLOGY Research and TreatMENT highlight the fundamental importance of oncological rehabilitation for children, adolescents, and young adults who have survived cancer. It is clear that oncological rehabilitation is highly important for a good start to the long-term care of cancer patients, and that further efforts are needed in order to combine these elements to an even greater extent.

\section{Disclosure Statement}

The authors declare no conflicts of interest.

\section{References}

1 Langer T, Grabow D, Steinmann D, Wörmann B, Calaminus G: Late effects and long-term follow-up after cancer in childhood. Oncol Res Treat 2017;40 DOI: $10.1159 / 000484936$.
Krauth KA: Family oriented rehabilitation (FOR) and rehabilitation of adolescents and young adults (AYA) in pediatric oncology. Oncol Res Treat 2017;40: DOI: 10.1159/000484609.

$\checkmark 3$ Rick O, Dauelsberg T, Kalusche-Bontemps E-M: Oncological rehabilitation. Oncol Res Treat 2017;40: DOI: $10.1159 / 000481709$.
4 Schmielau J, Rick O, Reuss-Borst M, Kalusche-Bontemps E-M, Steimann M: Rehabilitation of cancer survivors with long-term toxicities. Oncol Res Treat 2017; 40: DOI: $10.1159 / 000485187$

5 Seifart U, Schmielau J: Return to work of cancer survivors. Oncol Res Treat 2017;40: DOI: 10.1159/000485079.

\section{KARGER} () 2017 S. Karger GmbH, Freiburg 